\title{
EDITORIAL \\ Is the use of 5-ALA in endoscopic skull base surgery truly limited or in need of more refined evaluation?
}

\author{
Pablo F. Recinos, MD \\ Department of Neurological Surgery, Neurological Institute, Cleveland Clinic; and Minimally Invasive Cranial Base and Pituitary \\ Surgery Program, Rose Ella Burkhardt Brain Tumor and Neuro-Oncology Center, Neurological Institute, Cleveland Clinic, \\ Cleveland, Ohio
}

I $\mathrm{N}$ this issue of the Journal of Neurosurgery, Micko et al. evaluated the use of 5-aminolevulinic acid (5-ALA) optical fluorescence in 28 patients who underwent tumor resection via an endoscopic skull base approach. ${ }^{1}$ Over half of the patients had pituitary adenomas (54\%) and the remaining patients had other common sellar/suprasellar pathologies, including meningiomas, craniopharyngiomas, and Rathke's cleft cyst; 3 patients had skull base malignancies. Ultimately the authors found limited, if any, utility for use of 5-ALA fluorescence in endoscopic skull base surgeries, leaving a possible exception for meningiomas.

Various visualization techniques have been proposed for maximizing tumor cytoreduction in neurosurgical oncology. These include the use of 5-ALA fluorescence in the visible light spectrum, the use of indocyanine green in the near-infrared spectrum, the use of intraoperative ultrasonography, and the use of intraoperative MRI. By safely maximizing the extent of resection, potential goals include decreasing the likelihood of tumor recurrence and the need for other adjuvant treatment modalities (e.g., radiation). In addition, improved visualization can help avoid damage to eloquent tissue, such as the optic nerves or hypothalamus. Improved visualization can also lead to more aggressive yet still safe resection of tumor, which on initial inspection may appear indistinguishable from normal tissue.

Neoplastic cells have been found to accumulate excess protoporphyrin IX (PpIX) after systemic administration of 5-ALA. In turn, the accumulated PpIX produces a red fluorescence in the visible spectrum (wavelength $=$ $635 \mathrm{~nm}$ ) when it is viewed under blue light (wavelength $=440 \mathrm{~nm}$ ). ${ }^{2}$ Clinically, the use of 5-ALA was shown in a randomized controlled trial by Stummer and colleagues to significantly improve extent of resection rates for highgrade gliomas. ${ }^{3}$ Since then, its use has expanded to include benign tumors. For pituitary adenomas, the experience with fluorescence after 5-ALA administration has been variable and thus its clinical utility has been questioned. ${ }^{4}$ In the current study, pituitary adenomas did not show any fluorescence and thus this experience gives further weight to the argument favoring no role of 5-ALA in pituitary tumor surgery. ${ }^{1}$ For meningiomas, however, two studies have reported high degrees of fluorescence with 5-ALA, ranging from $91 \%$ to $94 \% .^{5,6} \mathrm{In}$ contrast, this is where the authors showed a significantly lower rate of fluorescence $(25 \%)$, albeit with a limited number of meningioma patients $(n=4)$. The discrepancy of the findings of this study with that of prior studies showing avid fluorescence in meningiomas after 5-ALA administration raises several interesting questions to guide future investigations.

First, the kinetics of 5-ALA in different tumor subtypes, and by extension the timing of 5-ALA administration, is worth considering. Recently, Kaneko and coworkers ${ }^{7}$ examined the in vivo kinetics of PpIX after administration of 5-ALA in meningiomas and glioblastomas. They noted that fluorescence intensity peaked 7-8 hours after 5-ALA administration. In addition, meningiomas actually displayed a higher fluorescence intensity compared to that of glioblastomas. The kinetics of PpIX were also different, as it appeared to clear faster in meningiomas compared to glioblastomas. Interestingly, there was no difference in fluorescence intensity between benign and atypical meningiomas. ${ }^{7}$ In the current study by Micko et al., patients received 5-ALA 2-4 hours prior to induction of anesthesia, with a median time to evaluation of 5.7 hours. ${ }^{1}$ This certainly appears to be consistent with the reported peak time for meningioma fluorescence. However, it is important to consider that the kinetics of PpIX may be significantly different in other tumor subtypes. Notably, Goryaynov et al. reported other tumor subtypes that were treated via an endoscopic endonasal approach (EEA) that were not reported in the current study: specifically, chordoma, neurinoma, and inverted papilloma were reported and did fluoresce after 5-ALA administration. ${ }^{5}$ This may 
warrant further consideration in future 5-ALA fluorescence studies for tumor subtypes that do not have welldefined PpIX kinetics.

Second, the meningiomas in this case were presumably all arising in the anterior skull base (e.g., tuberculum sellae, planum sphenoidale, etc.). In contrast, prior studies that examined 5-ALA fluorescence in meningiomas included tumors in all locations, not just the skull base. However, it would seem unlikely that location or histological subtype alone would result in a substantial difference in fluorescence. Although the skull base location does tend to favor benign meningioma grades, it has been noted that there is no significant difference in fluorescence patterns after 5-ALA administration between benign and atypical meningiomas. ${ }^{7,8}$ Nonetheless, more robust reporting of the specific meningioma site of origin would seem in order for precise comparisons.

Lastly, the authors raised an important point of whether coagulation of the dural layer prior to dural opening impacted the fluorescence pattern after 5-ALA administration. Certainly, devascularization is an early technical step performed routinely in meningioma surgery. In EEAs it is not only an early step, but it is an unavoidable step prior to starting the meningioma resection. Interestingly, in the subgroup of patients with meningiomas treated via an EEA, Goryaynov et al. reported that $75 \%$ demonstrated fluorescence after administration of 5-ALA compared to $25 \%$ in this study. ${ }^{1,5}$ Is this simply a chance finding or one that is reflective of substantial changes that occur in the meningioma after extensive coagulation, such that the accumulated PpIX is fundamentally altered so it no longer fluoresces? Was the timing of when the blue-light filter was activated relative to the timing of dural coagulation significant? We are left wondering.

The authors should be commended for their contribution here. However, in the end, I believe this study has raised more questions than answers. Given that there is still both a need and desire to improve adjunct visualization techniques, it remains to be seen whether the story of 5-ALA is indeed finished in endoscopic skull base surgery or just beginning to be refined.

https://thejns.org/doi/abs/10.3171/2020.7.JNS201870

\section{References}

1. Micko A, Rapoport BI, Youngerman BE, et al. Limited utility of 5-ALA optical fluorescence in endoscopic endonasal skull base surgery: a multicenter retrospective study. J Neurosurg. Published online October 30, 2020. doi:10.3171/2020.5.JNS201171

2. Missios S, Abbassy M, Vogelbaum MA, Recinos PF. Use of image fluorescence in the resection of gliomas. Curr Surg Rep. 2015;3:76.

3. Stummer W, Pichlmeier U, Meinel T, et al. Fluorescenceguided surgery with 5-aminolevulinic acid for resection of malignant glioma: a randomised controlled multicentre phase III trial. Lancet Oncol. 2006;7(5):392-401.

4. Chang SW, Donoho DA, Zada G. Use of optical fluorescence agents during surgery for pituitary adenomas: current state of the field. J Neurooncol. 2019;141(3):585-593.

5. Goryaynov SA, Okhlopkov VA, Golbin DA, et al. Fluorescence diagnosis in neurooncology: retrospective analysis of 653 cases. Front Oncol. 2019;9:830.
6. Millesi M, Kiesel B, Mischkulnig M, et al. Analysis of the surgical benefits of 5-ALA-induced fluorescence in intracranial meningiomas: experience in 204 meningiomas. $J$ Neurosurg. 2016;125(6):1408-1419.

7. Kaneko S, Brokinkel B, Molina ES, et al. Real-time in vivo kinetics of protoporphyrin IX after administration of 5-aminolevulinic acid in meningiomas and comparative analyses with glioblastomas. Acta Neurochir (Wien). 2020;162(9):2197-2202.

8. Lee JH, Sade B, Choi E, et al. Meningothelioma as the predominant histological subtype of midline skull base and spinal meningioma. J Neurosurg. 2006;105(1):60-64.

\section{Disclosures}

The author reports no conflict of interest.

\section{Correspondence}

Pablo F. Recinos: recinop@ccf.org.

INCLUDE WHEN CITING

Published online October 30, 2020; DOI: 10.3171/2020.7.JNS201870.

\section{Response}

\section{Gabriel Zada, MD}

Department of Neurosurgery, Keck School of Medicine, University of Southern California, Los Angeles, California

We read with interest and appreciate Dr. Recinos' comments on our recent investigation of 5-ALA fluorescence status in a variety of skull base pathologies using a bluelight endoscope.

In our study, we showed that during endoscopic transsphenoidal resection of pituitary adenomas, there was no benefit in using 5-ALA as a fluorescence agent. ${ }^{1}$

However, we agree that 5-ALA may offer a certain value in the resection of tuberculum sellae or planum sphenoidale meningiomas, with 1 of 4 patients showing strong but heterogenous fluorescence. In a study by Millesi et al., $280 \%$ of tuberculum sellae meningioma cases showed a vague fluorescence within tumor tissue. While Dr. Recinos has postulated that extensive cauterization and devascularization of the dural layer prior to opening may have resulted in a high rate of negative 5-ALA fluorescence in our multicenter study, the mechanism by which devascularization of a meningioma would decrease fluorescence is not elucidated. Indeed, operations on convexity meningiomas also devascularize the tumors during dural opening and fluorescence is not compromised in this situation. Why would skull base cases be any different? Another possible influence, as indicated by Dr. Recinos, is that the timing of optimal 5-ALA administration may be a contributing factor; due to consistency in the timing of administration in both institutions, however, it is rather unlikely that this is the main cause of the variance in fluorescence of meningiomas seen in our study.

Furthermore, the examination of other rare tumors of the skull base, such as craniopharyngiomas, Rathke's cleft cysts, plasmacytomas, esthesioneuroblastomas, and sinonasal squamous cell carcinomas, resulted in negative 5-ALA fluorescence in our case series. Chordomas, chondrosarcomas, neurinomas, and inverted papillomas were not assessed in our study, thereby still allowing the pos- 
sibility of using a blue-light endoscope for in vivo optical tissue imaging of these tumors. However, positive fluorescence ( 1 of each cases of these pathologies) was reported by Goryaynov et al.; ${ }^{3}$ therefore, in these and other less common skull base pathologies not examined in this report, investigation with larger case numbers may still be warranted. Overall, we agree with Dr. Recinos' sentiment that further exploratory work in this arena should be performed to optimize the indications for blue-light fluorescence during endoscopic skull base surgery, especially as it pertains to modifications in timing of administration of 5-ALA and identifying other pathologies that may benefit from this adjunctive technique.

\section{References}

1. Marbacher S, Klinger E, Schwyzer L, et al. Use of fluorescence to guide resection or biopsy of primary brain tumors and brain metastases. Neurosurg Focus. 2014;36(2):E10.

2. Millesi M, Kiesel B, Mischkulnig M, et al. Analysis of the surgical benefits of 5-ALA-induced fluorescence in intracranial meningiomas: experience in 204 meningiomas. $J$ Neurosurg. 2016;125(6):1408-1419.

3. Goryaynov SA, Okhlopkov VA, Golbin DA, et al. Fluorescence diagnosis in neurooncology: retrospective analysis of 653 cases. Front Oncol. 2019;9:830.

\section{INCLUDE WHEN CITING}

Published online October 30, 2020; DOI: 10.3171/2020.7.JNS202669. 